\title{
ELECTRIC DEVICE WITH LINEAR MOTOR FOR ROBOT-WEEDING
}

\author{
Alexander Lysakov, Gennady Nikitenko, Evgeny Konoplev, Ekaterina Sergienko \\ Stavropol State Agrarian University, Russia \\ s_lysakov@mail.ru, nikitenko_gv@mail.ru,konoplev82@mail.ru, nikitenko_eg@mail.ru
}

\begin{abstract}
The goal of decreasing herbicide usage has so far focused on reducing the herbicide dosage or replacing chemical weed control by hoeing and harrowing. The conventional weed control strategy is to apply the same dose rate of herbicide or the same intensity of physical treatments to the whole field. This strategy may be changed in the coming years, because several investigations have shown that site specific weed management can reduce herbicide usage significantly. One of the promising technologies within site specific weed management is robotic weeding. Today the most challenging problem is still to remove weeds within the rows and close to crop plants. So far, no commercial selective mechanical methods have been developed for the intra-row or close-to-crop areas, thus, $10 \%$ to $20 \%$ of a field has to be controlled by band spraying or manual weeding. Novel mechanical or chemical weeding methods therefore have to be developed in order to achieve further reduction in the herbicide usage. A new idea is to combine computer vision and seed mapping to obtain improved overall accuracy, reliability and efficiency in geo-referencing the crop and weed seedlings. Unfortunately, oscillating and rotating tillage devices cannot be used in the close-to-crop area due to the high risk of root or leaf damage of crop seedlings. Therefore, the close-to-crop area has to be treated with highly accurate operating tools. The most appropriate tool should avoid root damage and therefore operate above-ground (not tilling). Promising tools could be very small spinning blades, a laser or a picking device. An alternative method to physical weeding in the close-to-crop area could be the micro-spray system that deposits micro-volumes of a herbicide on single weed plants. In this paper, a design of highly accurate operating robot arm based on linear motor is presented.
\end{abstract}

Keywords: weed recognition, precision agriculture, linear motor.

\section{Introduction}

The agricultural industry is one of the most important sectors of the economy of any country. The list of agricultural products is extremely diverse: from vegetables and fruits supplied to the shelves of stores, mixed feeds intended for raising animals, to raw materials used in various industries. According to demographic studies, the population will increase to 9 billion people by 2050 , and to meet the increased demand, the production of the agricultural industry must increase by $25 \%$.

To achieve such indicators, the use of robotic technology becomes an important necessity. Today, human labor is becoming more and more expensive, and monotonous operations in uncomfortable positions are not able to give the expected quality results. The solution to this problem lies in the use of robotic technology by farmers in weed control, harvesting and field processing [1-2].

The use of robots allows to eliminate the loss of working time associated with absenteeism, illness, tardiness and other human factors. As a result of the introduction of robots, the annual fund of working time is released, which leads to additional production of products. It is possible to perform a wide range of operations very conveniently and quickly, thereby eliminating the fatigue of workers due to highintensity manual labor [3-4].

Increasing productivity and labor efficiency in agro-industrial production with the help of robotics can be achieved only on the principle of full automation of algorithmic operations in intellectual and industrial human activities with integrated automation of flexible production subsystems [5-6].

Robotic devices are used to eliminate weeds in the seed line between cultivated plants (intra-row), while weeds between seed lines (inter-row) are controlled by conventional cultivation methods [7].

Currently, robotic weeding machines use one of several means of destroying weeds, including mechanical, flame or herbicide spraying, as well as lasers [8-9].

The mechanical design consists of an end effector, manipulator, and gripper. Several factors must be considered in the design of the manipulator, including the task, economic efficiency, and required motions.

An end effector in an agricultural robot is the device found at the end of the robotic arm, used for various agricultural operations. The gripper is a grasping device that is used for harvesting the target crop. Design of the gripper is based on simplicity, low cost, and effectiveness. Thus, the design usually consists of two mechanical fingers that are able to move in synchrony when performing their task. 
Specifics of the design depend on the task that is being performed. For example, in a procedure that required plants to be cut for weeding, the gripper was equipped with a sharp blade.

The manipulator allows the gripper and end effector to navigate through their environment. The manipulator consists of four-bar parallel links that maintain the gripper's position and height. The manipulator also can utilize one, two, or three pneumatic actuators. Pneumatic actuators are motors, which produce linear and rotary motion by converting compressed air into energy. The pneumatic actuator is the most effective actuator for agricultural robots because of its high power-weight ratio. The most cost efficient design for the manipulator is the single actuator configuration, yet this is the least flexible option.

Also, the manipulator can use an alternating current (AC) asynchronous motor, and a direct current (DC) motor. These motors use torque, which requires an additional transmission mechanism in the form of a gearbox, which increases the weight of the manipulator. Also, the asynchronous motor and DC motor are not high-precision equipment, and when used in weeding, other plants may be damaged.

To eliminate these disadvantages, it is necessary to use a linear electric motor. A linear motor is an electric motor that has had its stator and rotor "unrolled", thus, instead of producing a torque (rotation), it produces a linear force along its length.

In linear motors, there are no transmission mechanisms, no conversion of rotation into linear motion, no coupling couplings, no belt drive and other transmission. Linear motors have high accuracy and movement dynamics. Also, linear motors have a simple design, they are reliable and durable.

The aim of the research was to simulate the electromagnetic field of a linear motor to obtain the maximum values of the magnetic field induction and magnetic field strength.

\section{Materials and methods}

During the experiment standard the following research methods were applied: a multifactorial experiment method, a statistical analysis, an adequacy test [10-11].

The simulation was performed in a software product. The software product consists of modules that allow you to analyze physical fields and obtain solutions to related interdisciplinary problems in the following types of analysis: the magnetic field of alternating currents; the magnetic field of direct currents and/or permanent magnets; the non-stationary magnetic field; the electrostatic field; the electric field of direct currents; the electric field of alternating currents; the non-stationary electric field; the stationary and non-stationary temperature field; mechanical stresses and elastic deformations.

The proposed models of magnetic fields are based on numerical methods of mathematical physics and the variational principle for the approximate solution of the Laplace and Poisson equations, which leads to the replacement of the solution of partial differential equations by minimizing the energy functional. The models are based on finite element and finite difference methods.

The problems of magnetostatics can be solved in linear and nonlinear formulations. The source of the field can be concentrated and distributed currents and current layers, permanent magnets, as well as external magnetic fields. In solving these problems, the Poisson equation is used for the vector magnetic potential $A$ ( $B=\operatorname{rot} A, B$ is the vector of magnetic induction). In the problems under consideration, the induction vector $B$ always lies in the plane of the model ( $x y$ or $z r$ ), and the current density vector $\mathrm{j}$ and the vector potential $A$ are perpendicular to it. Only the components $j_{z}$ and $A_{z}$ are different from zero in the plane-parallel case, or $j_{\theta}$ and $A_{\theta}$ in axisymmetric problems.

The capabilities of the software product allow to use not the entire model, but only half of it (due to the symmetric distribution of magnetic fields). Therefore, when calculating the parameters, only half of the model is shown, inverted by $90^{\circ}$ to the coordinate axis.

To simulate the electromagnetic field, three design options were developed for the stationary stator of a linear motor. For the first option, the placement of two coils on the stator is selected, for the second option, one coil is selected for the entire length of the stator body, for the third option, one coil is selected, located in the middle of the stator. For the simulation conditions, the voltage of $12 \mathrm{~V}$ and the necessary magnetic properties of the material for the manufacture of the stator coil were established. The main results of modeling the electromagnetic field in a stationary stator of a linear motor are presented in Figures 1-6. 


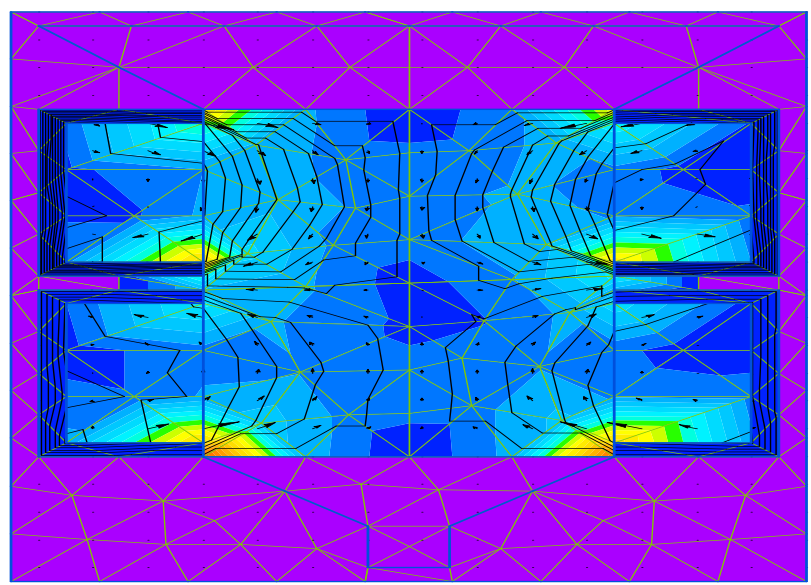

Magnetic field strength, $H, 10^{4} \mathrm{~A} \cdot \mathrm{m}^{-1}$

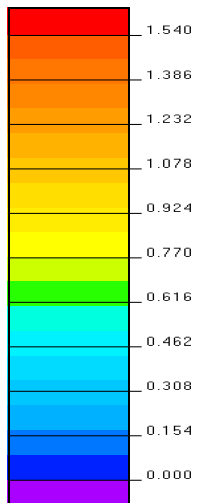

Fig. 1. Distribution of the magnetic field strength in the linear motor stator for option No. 1

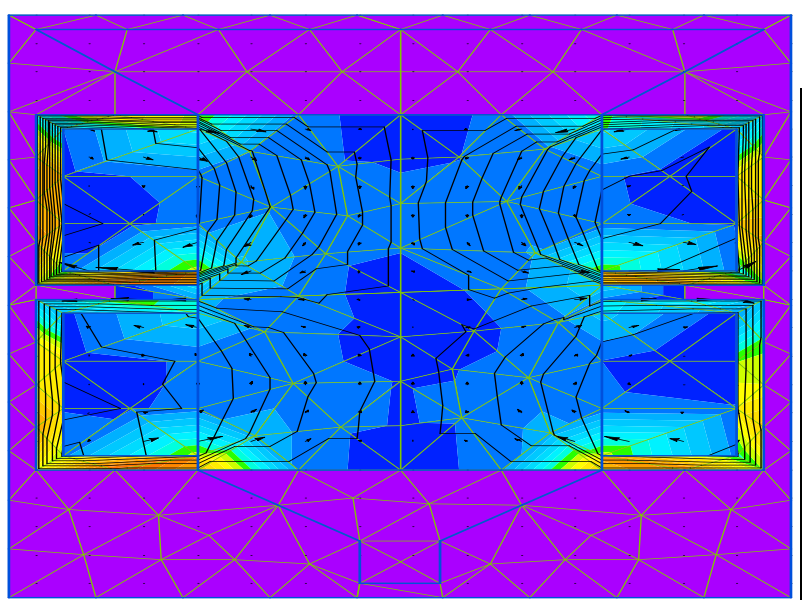

Magnetic induction,

$B, \mathrm{~T}$

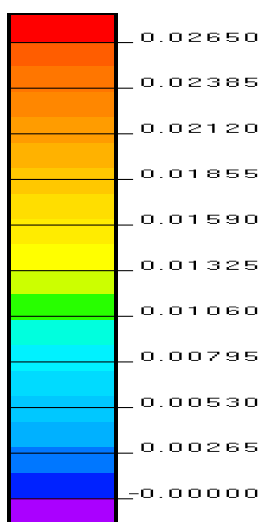

Fig. 2. Magnetic induction distribution in the linear motor stator for option No. 1

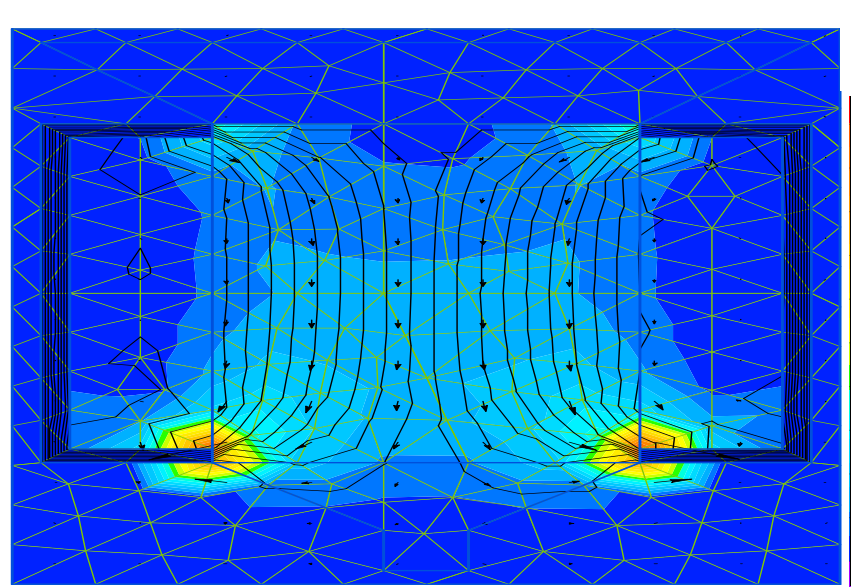

Magnetic field strength, $H, 10^{4} \mathrm{~A} \cdot \mathrm{m}^{-1}$

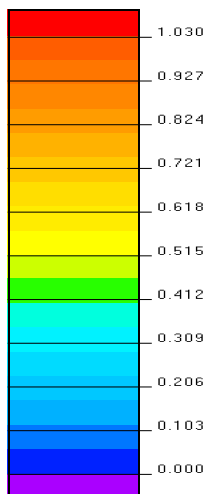

Fig. 3. Distribution of the magnetic field strength in the linear motor stator for option No. 2 

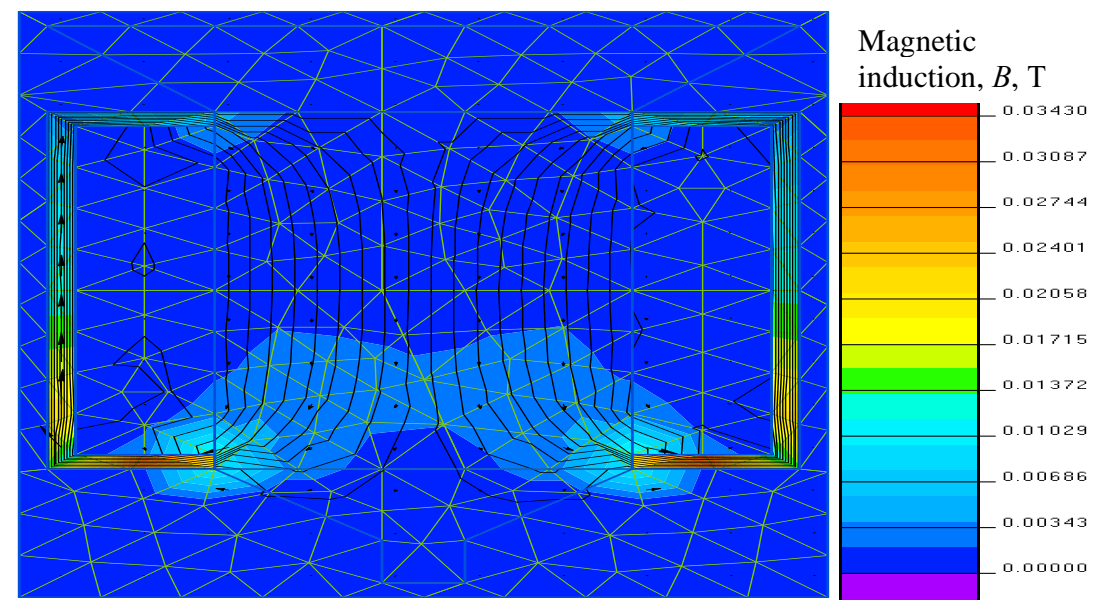

Fig. 4. Magnetic induction distribution in the linear motor stator for option No. 2

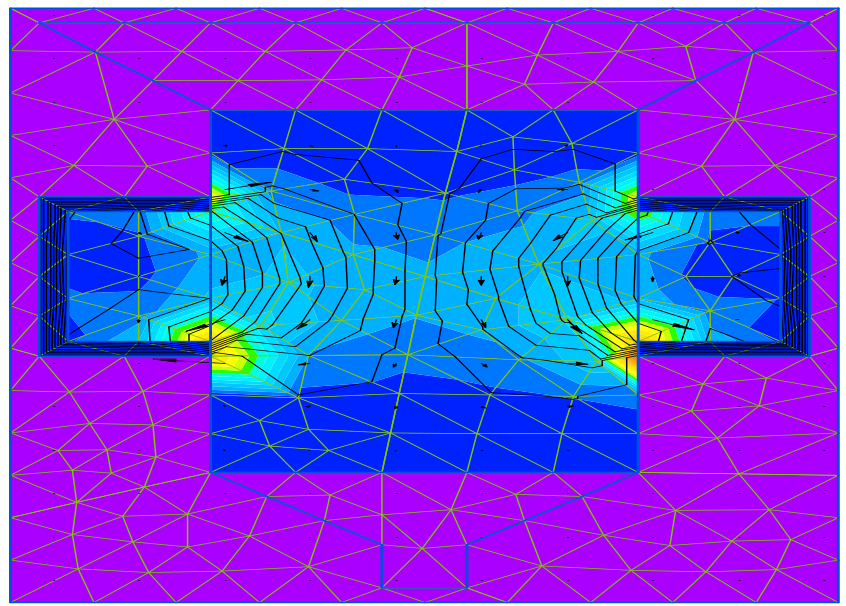

Magnetic field strength, $H, 10^{4} \mathrm{~A} \cdot \mathrm{m}^{-1}$

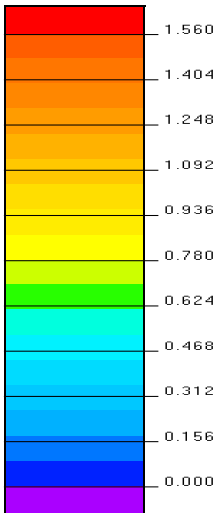

Fig. 5. Distribution of the magnetic field strength in the linear motor stator for option No. 3

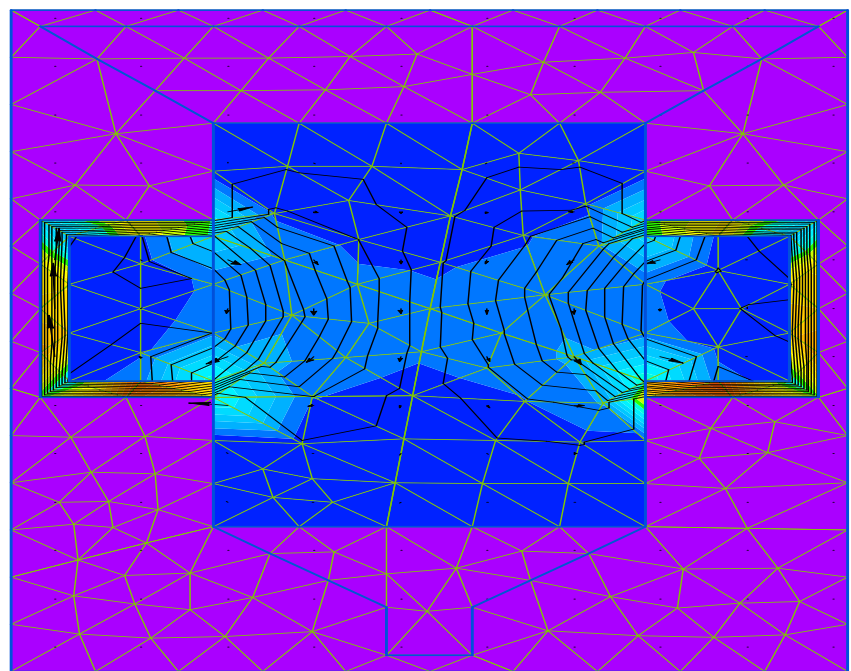

\section{Magnetic}

induction, $B$, T

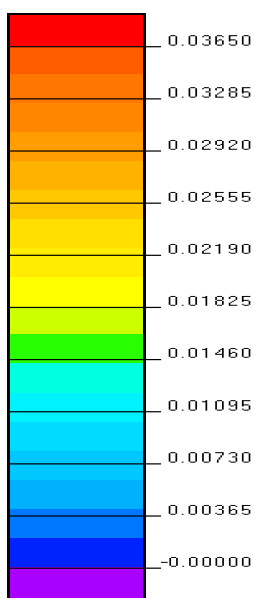

Fig. 6. Magnetic induction distribution in the linear motor stator for option No. 3

\section{Results and discussion}

When determining the parameters of the electromagnetic field for a linear motor having two coils, the operating modes and the picture of the electromagnetic field were also modeled, in which the 
currents in the coils were directed in opposite directions, or in opposite directions, and the operating modes with a ferromagnetic core, without a ferromagnetic core, with the use of diamagnetic inserts were also studied.

The maximum value of the magnetic flux equal to $2.3 \mu \mathrm{Wb}$ is observed for option 3 . The maximum value of the magnetic field strength equal to $2641.7 \mathrm{~A} \cdot \mathrm{m}^{-1}$ is observed for option 1 . The maximum value of the magnetic induction equal to $3.3 \mathrm{mT}$ is observed for option 1. The main simulation results for various fixed stator designs of a linear motor are shown in Table 1.

Analyzing the results of computer modeling of magnetic fields and the picture of the distribution of the magnetic field strength and magnetic induction over the linear motor stator, it can be concluded that the linear motor option No. 1, in comparison with options No. 2 and No. 3, is the most effective and profitable for moving the linear motor rotor, because the distribution of the strength and magnetic induction in the working area of the stator is uniform and meets the conditions necessary for accurate movement of the rotor.

Table 1

Results of computer simulation of magnetic field parameters

\begin{tabular}{|c|cc|c|c|c|c|}
\hline \multirow{4}{*}{$\begin{array}{c}\text { Option } \\
\text { number }\end{array}$} & \multicolumn{5}{|c|}{ Magnetic field parameters } \\
\cline { 2 - 7 } & $\begin{array}{c}\text { Magnetic } \\
\text { flux, } \\
\boldsymbol{\Phi}, \mathbf{W b}\end{array}$ & $\begin{array}{c}\text { Magnetic } \\
\text { induction, } \\
\boldsymbol{B}, \mathbf{T}\end{array}$ & $\begin{array}{c}\text { Magnetic } \\
\text { field } \\
\text { strength, } \\
\boldsymbol{H}, \mathbf{A} \cdot \mathbf{m}^{-1}\end{array}$ & $\begin{array}{c}\text { Magnetic } \\
\text { flux, } \\
\boldsymbol{\Phi}, \mathbf{W b}\end{array}$ & $\begin{array}{c}\text { Magnetic } \\
\text { induction, } \\
\boldsymbol{B}, \mathbf{T}\end{array}$ & $\begin{array}{c}\text { Magnetic } \\
\text { field } \\
\text { strength, } \\
\boldsymbol{H}, \mathbf{A} \cdot \mathbf{m}^{-\mathbf{1}}\end{array}$ \\
\hline 1 & $9.2304 \cdot 10^{-7}$ & $23.474 \cdot 10^{-4}$ & 1868 & $1.3054 \cdot 10^{-6}$ & $33.197 \cdot 10^{-4}$ & 2641.7 \\
\hline 2 & $1.5349 \cdot 10^{-7}$ & $16.148 \cdot 10^{-4}$ & 1285 & $2.1706 \cdot 10^{-7}$ & $22.836 \cdot 10^{-4}$ & 1817.2 \\
\hline 3 & $16.797 \cdot 10^{-7}$ & $1.6284 \cdot 10^{-4}$ & 129.58 & $2.3754 \cdot 10^{-6}$ & $2.3015 \cdot 10^{-4}$ & 183.15 \\
\hline
\end{tabular}

\section{Conclusions}

1. The use of a linear motor in the robot's manipulator for weeding plants is an accurate and safe way to destroy weeds.

2. For computer simulation, three versions of the stationary stator of a linear motor were created.

3. As a result of computer simulation option 1 is selected, which has the following parameters: magnetic flux equal to $1.3 \mu \mathrm{Wb}$, magnetic field strength equal to $2641.7 \mathrm{~A} \cdot \mathrm{m}^{-1}$, magnetic induction equal to $3.3 \mathrm{mT}$.

4. Analyzing the results obtained, we can conclude that the opposite direction of the currents in the coil increases the magnetic field at the same current density. This makes it possible to save the energy consumed, so in the future this method will be used as the most preferred.

5. The separation of the stator area into several parts by means of two magnetizing coils makes the distribution of the magnetic field uniform, efficient and easier to adjust, which is very important in the case of smooth and precise movement of the linear motor rotor.

\section{References}

[1] Raja R. Nguyen T. T., Slaughter D. etc. Real-time weed-crop classification and localisation technique for robotic weed control in lettuce. Biosystems Engineering, vol. 192, 2020, pp. 257-274.

[2] Hansen K.D., Garcia-Ruiz F., Kazmi W., Bisgaar M. etc. Multi-Agent Planning for Coordinated Robotic Weed Killing. IFAC Proceedings Volumes, vol. 46, Issue 10, 2013, pp. 217-224.

[3] Slaughter D., Giles D.K., Downey D. Autonomous robotic weed control systems: A review. Computers and Electronics in Agriculture, vol. 61, 2008, pp. 63-78.

[4] Sabanci K., Aydin C. Smart Robotic Weed Control System for Sugar Beet. Journal of Agricultural Science and Technology, vol. 19, 2017, pp. 78-83.

[5] Chen L., Kaewkorn S., He L., Zhang Q, etc. Design and Evaluation of a Levelling System for a Weeding Robot. IFAC-PapersOnLine, vol. 49, Issue 16, 2016, pp. 299-304.

[6] Chen L., Karkee M., He L., Wei Y., and Zhang Q. Evaluation of a Leveling System for a Weeding Robot under Field Condition. IFAC-PapersOnLine, vol. 51, Issue 17, 2018, pp. 368-373. 
[7] Pérez-Ruíz M., Slaughter D., Fathallah F.A., Gliever C. etc. Co-robotic intra-row weed control system. Biosystems Engineering, vol. 126, 2014, pp. 45-55.

[8] Kaierle S., Marx C. Find and irritate - laser used for weed control. Laser Technik Journal, vol. 10, 2013, pp. 44-47.

[9] Xiong Y., Ge Y., Liang Y., and Blackmore S. Development of a prototype robot and fast pathplanning algorithm for static laser weeding. Computers and Electronics in Agriculture, vol. 142, part B, 2017, pp. 494-503.

[10] Lysakov A., Tarasov Y. Effect of magnetic field of neodymium ( $\mathrm{NdFeB}$ ) magnet on storage quality of potatoes. Proceedings of International conference "Engineering for Rural Development", 2019, pp. 609-612.

[11] Lysakov A., Nikitenko G., Konoplev E., Grinchenko V. Method to increase cleaning degree of electrostatic air cleaner. Proceedings of International conference "Engineering for Rural Development", 2018, pp. 554-559. 